This item was submitted to Loughborough's Research Repository by the author.

Items in Figshare are protected by copyright, with all rights reserved, unless otherwise indicated.

\title{
Experimental investigation of spectral effects on amorphous silicon solar cells in outdoor operation
}

PLEASE CITE THE PUBLISHED VERSION

http://dx.doi.org/10.1109/PVSC.2002.1190807

PUBLISHER

(C) IEEE

VERSION

VoR (Version of Record)

LICENCE

CC BY-NC-ND 4.0

\section{REPOSITORY RECORD}

Gottschalg, Ralph, Thomas R. Betts, David Infield, and Michael J. Kearney. 2019. "Experimental Investigation of Spectral Effects on Amorphous Silicon Solar Cells in Outdoor Operation”. figshare.

https://hdl.handle.net/2134/8219. 
This item was submitted to Loughborough's Institutional Repository (https://dspace.lboro.ac.uk/) by the author and is made available under the following Creative Commons Licence conditions.

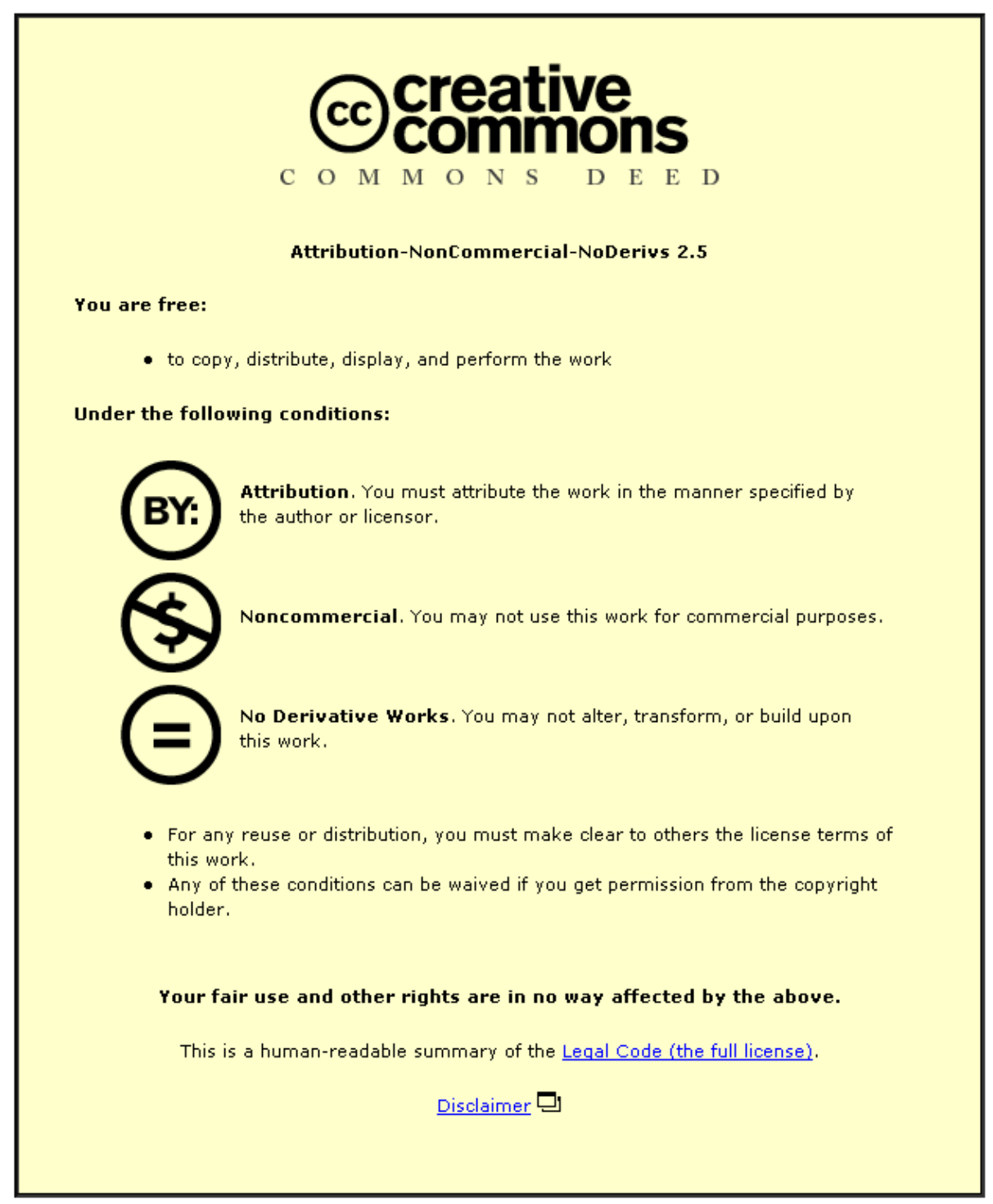

For the full text of this licence, please go to: http://creativecommons.org/licenses/by-nc-nd/2.5/ 


\title{
EXPERIMENTAL INVESTIGATION OF SPECTRAL EFFECTS ON AMORPHOUS SILICON SOLAR CELLS IN OUTDOOR OPERATION
}

\author{
R. Gottschalg, T.R. Betts, D.G. Infield, M.J. Kearney \\ Centre for Renewable Energy Systems Technology, Department of Electronic and Electrical Engineering, \\ Loughborough University, Loughborough, Leicestershire, LE11 3TU, UK
}

\begin{abstract}
The effect of varying spectrum on PV output is often underestimated in the case of amorphous silicon photovoltaic devices. This paper gives an indication of the order of magnitude of the seasonal variation of the useful irradiance in a maritime climate and also shows that this will involve a direct change in efficiency. This can be expected to be in the range of 15 percent around the annual average, thus explaining the seasonal performance. The spectral effects are investigated by distinguishing between a primary and a secondary effect. The primary effect is dependent on the availability of useful spectral irradiance while the secondary effect depends also on the spectral composition of the light in the useful range. It is shown that the secondary effect is especially significant for double junction devices.
\end{abstract}

\section{INTRODUCTION}

Amorphous silicon (a-Si) photovoltaic arrays are often shown to have a very good performance ratio. This high $\mathrm{kWh}$ per $\mathrm{kW}_{\mathrm{p}}$ is attributed to the seasonal performance pattern of these devices. Contrary to other devices, a-Si devices perform better in summer than in winter. This can be attributed either to seasonal annealing of the Staebler Wronski effect or to spectral effects. The importance of either effect will be location dependent. However, the spectrum is known to have a significant influence at all locations. For Loughborough, UK, we found that we can, based on an investigation of the available spectral irradiation, attribute the seasonal variation nearly exclusively to spectral effects [1].

Spectral effects occur since the spectrum experienced by devices during outdoor operation is not the AM1.5 spectrum normally used for indoor calibration. In fact, the incident spectrum is strongly dependent on air mass and weather, as shown e.g. in [2]. Thus it is important to understand the effect of varying spectrum on the operation of different devices. This paper demonstrates the effect of spectral variations on the performance parameters of single and double junction devices produced by RWE Solar, Division Phototronics. It is demonstrated that there are two different effects observable. First, there is a direct relation to the incident spectral irradiance in the useful spectral range. This effect is termed primary spec- tral effect in what follows. Having removed this, there is a further effect that depends also on the spectral composition of the irradiance received in the useful range. This effect is termed the secondary spectral effect.

\section{EXPERIMENTAL ARRANGEMENT}

In order to enable an investigation of spectral effects, a comprehensive measurement system has been set-up at the Centre for Renewable Energy Systems Technology (CREST). The system combines data from a custom made spectroradiometer, two Kipp\&Zonen CM11 pyranometers, and the I-V characteristic and temperature of the measured devices. The I- $V$ characteristic is measured using a switching circuit developed in-house to access the different devices, the actual scanning is performed using a Keithley 2420 source-measure unit (SMU) through a 4 wire connection. Measurements were carried out every 10 minutes, and consist of the incident spectral irradiance in the range from $300 \mathrm{~nm}$ to 1700 , the standard environmental data and the device measurements. The environmental data (including spectral information) has been recorded since May 1998.

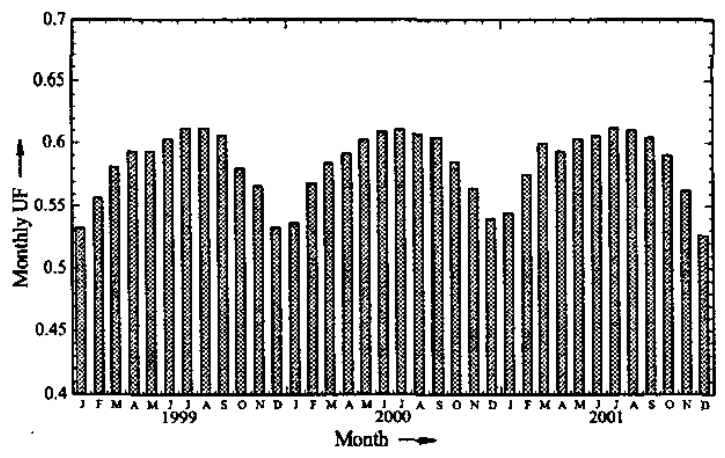

Fig. 1. Seasonal variation of the monthly UF for a-Si devices over a three year period.

Each device measurement is preceded by a measurement of the global irradiance and the device temperature. The I-V scan of 200 points is then followed by a second measurement of the global irradiance in order to guarantee stability during the measurements. 
In the following, the difference in considering the spectrally useful irradiance and the total energy in the incident spectra is investigated. In order to avoid confusion with global irradiance (i.e. the sum of beam, diffuse and albedo), the latter is termed total irradiance.

The measurements presented in this paper were taken in the period from October 1999 to March 2000 using single and double junction devices. The devices are identical in their dimensions, the only difference is the number of junctions. These devices were sealed at CREST and installed in the outdoor measurement system. Measurements with less than $10 \mathrm{~W} / \mathrm{m}^{2}$ were neglected, as there was a significant noise in the spectroradiometer measurements for these low intensities. Some noise could not be avoided, as the scanning of the spectral range takes about 2 min. This was minimised by analysing the measurements taken at the beginning and the end of the $\mathrm{I}-\mathrm{V}$ scans of all devices (including those not analysed here) and time points with scatter of more than $5 \%$ in these measurements were rejected.

\section{USEFUL FRACTION}

It is necessary to have a measure of 'blueness' of the light for the analysis of the measurements. It was found useful to define a useful fraction (UF) as the energy in the useful range of the device to the total energy in the incident irradiance. This works well for devices with a limited spectral response, such as a-Si devices. The useful range for a-Si is taken throughout this paper to be $300-780 \mathrm{~nm}$, as supported by the fiterature [3]. The maximal wavelength considered for the calculation of the total irradiance is, due to the limitations of the spectroradiometer, 1700 $\mathrm{nm}$. This measure has a direct correlation with the performance of a device. It also can be used for monthly energy sums, as done in Fig. 1, to investigate the expected seasonal variation of device performance.

It is apparent from Fig. 1 that there is a clear variation of the useful fraction in the course of a year. It varies $+6 \%$ to $-9 \%$ with respect to the average of all measurements between summer and winter time respectively. This is sufficient to explain the seasonal variations reported for a$\mathrm{Si}$. This effect will occur whenever the spectral response of the device monitoring the incident irradiance and the device actually monitored is not matched.

\section{RESULTS AND DISCUSSION}

The variation of the incident spectrum will affect the device, as illustrated using the example of the short circuit current of a single junction device in Fig. 2. When simply dividing the short circuit current by the total irradiance as measured with the pyranometer and plotting versus the irradiance, a functional relationship seems to exist.

The reduction in scatter at higher irradiances in Fig. 2 , is expected, as these typically occur in low air mass, low cloud cover conditions. Thus no larger spectral variation is expected. The spectraf variations towards lower irradiances are much more significant, as these can be due to higher air mass of the incident irradiance or higher cloud cover. The first will alter the UF towards the red end of the spectrum while the latter causes a shift towards the blue end of the spectrum. This effect can be removed by using the spectrally useful irradiance for the calculation of the $I_{\mathrm{SC}}$ over $\mathrm{G}$ ratio, as done in all the following plots. We term this effect the primary spectral effect, as it depends on the availability of spectrally useful irradiance.

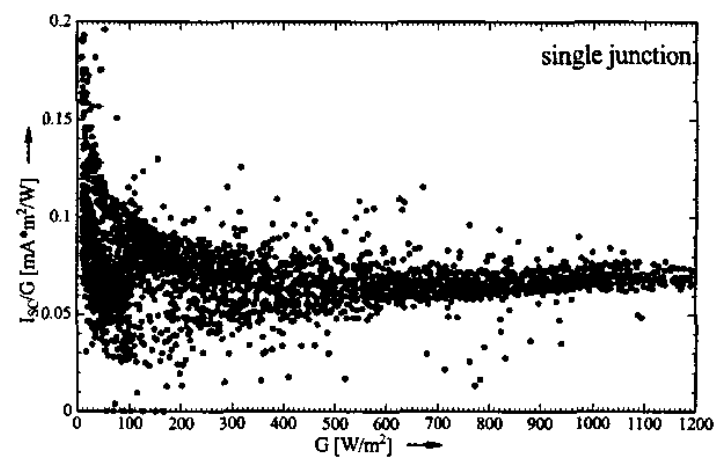

Fig. 2. Dependence of the $I_{S c}$ on total irradiance.

All values will be presented with respect to the average of all measurements, as this shows the variation more clearly. Plotting these normalised values against the useful fraction allows an analysis of the importance of the spectral composition on the device performance. This effect is termed the secondary spectral effect.

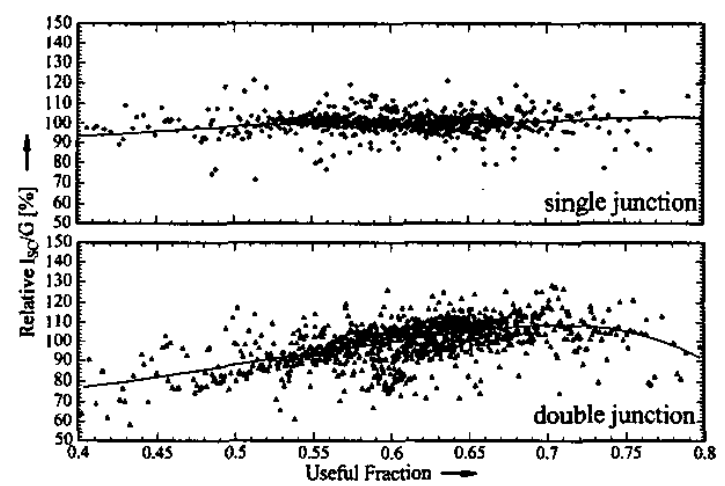

Fig. 3. Influence of the UF on the spectral Isc.

The spectral effects are most noticeable at short circuit conditions. The data is presented in Fig. 3 for a single junction device and a double junction device in the upper and lower plots respectively. Only one in ten points was plotted in order to make the fitted function visible. The single junction device is described well with a linear correlation. There is only a minor secondary spectral effect, which is mainly noticeable for very red and very blue spectra. The double junction, on the other hand, cannot be described so easily with a linear fit, a fourth order polynomial was used here instead. This was done purely because an increase/decrease in the order did not affect the correlation coefficient significantly. It is apparent that the secondary effect is more pronounced for a double junction. This could be expected, as the cell is optimised for AM1.5 spectra, which corresponds to a UF of 0.64 . The cells in a stack are connected in series and, according to Kirchhoff's law, the current in a series connection must be 
identical. Thus the maximum for mid range UFs is explained as well as the reduction towards the red and the blue end of the UF. The effect of the spectrum is also visible for the current at the maximum power point $I_{M P p}$, as shown in Fig. 4.

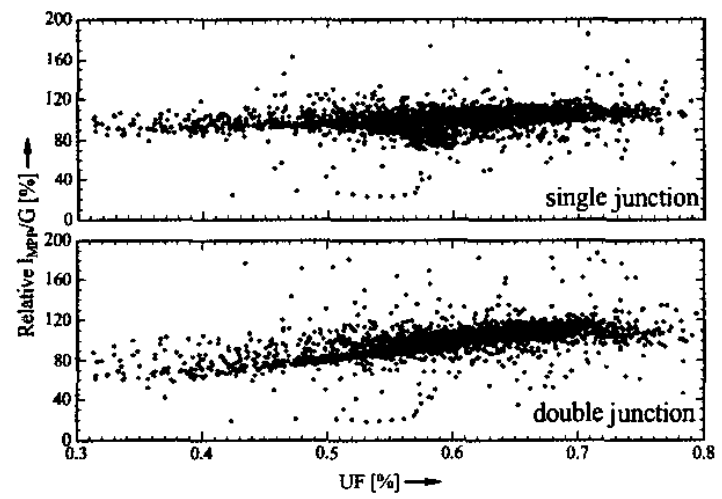

Fig. 4. Influence of UF on $I_{\text {MPP. }}$

The effect of the spectral variations on $I_{\text {MPP }}$ exhibits a small increase in scatter at UFs around 0.58 . Here other environmental effects begin to appear. The double junction, which exhibits a much more stable performance during this experiment [1], does not show these effects to a significant extent. These additional effects are the magnitude of the irradiance, the operating temperature and the age of the device. Choosing winter months helps to reduce the influence degradation/recovery of StaeblerWronski degradation, but does not exclude this completely. The shape of the dependence on the blueness of the light is very similar to the short circuit current.

There are further influences of the spectrum on the performance of a-Si devices. The fill factor FF is plotted versus the blueness in Fig. 5. The increase of scatter for mid-range UFs is due to progressing degradation and thus is stronger for single junctions than for double junctions. The effect of other environmental factors is much stronger for the FF, as any degradation in the shunt or series resistance and the lifetime of the charge carriers will affect the voltages as well as the currents and thus a stronger effect is observed.

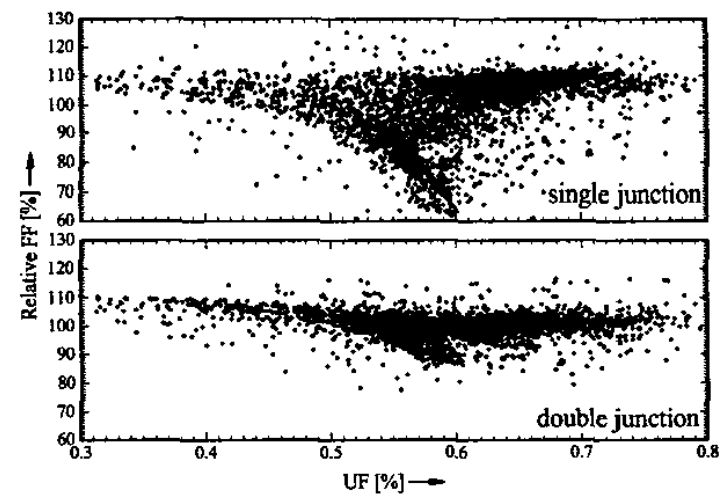

Fig. 5. Influence of UF on FF.
The behaviour of the FF shown in Fig. 5 also indicates a further difference between single and double junction devices. The single junction appears to exhibit an increase in FF with increasing blueness of the light (when neglecting the increased scatter for mid range UFs), while the double junction exhibits a reduction in the FF. There is a noticeable effect, but it is by no means as pronounced as reported by Rüther et al. [4]. This could be due to the use of different materials, however.

The FF is defined as $\left.\right|_{M P P} V_{M P P} V_{O C}{ }^{-1} I_{S C}{ }^{-1}$, thus the effect on the current should ameliorate itself to a large extent. The remaining effect should then be due to the voltages, as indeed depicted in Fig. 6, where the ratio of these voltages in investigated, as there is no apparent way to investigate these directly.

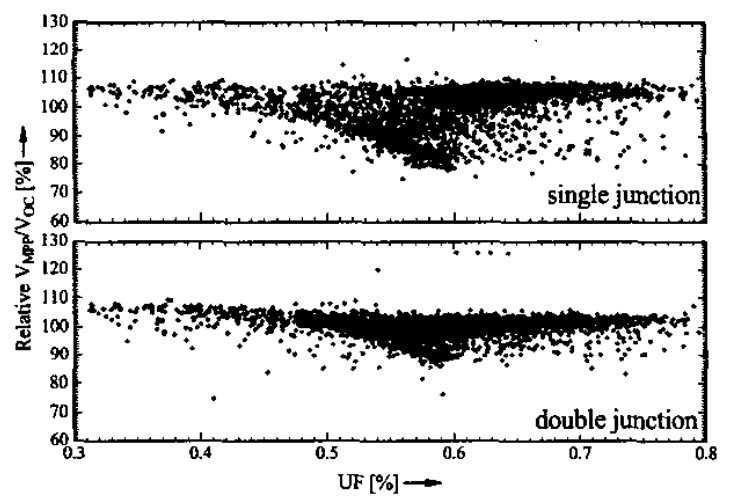

Fig. 6. Influence of the UF on the $\mathrm{V}_{\mathrm{MPP}} N_{\mathrm{OC}}$ ratio.

The ratios for the single and the double junction device show a similar functional relationship as the FF. Again, there is a significant increase in scatter for mid range conditions and as explained above, the variation of other environmental factors is higher in this region. It is interesting to note that the double junction exhibits a slight reduction in the generated voltage ratios for bluer light, while the single junction exhibits a slight increase.

Having investigated all parameters separately, it is logical to investigate the variation of the efficiency with blueness of the light as done in Fig. 7.

The efficiency variation of a single junction does not show a strong functional relationship with blueness of the light. This can be expected, as there should not be a significant secondary spectral effect for these devices. The primary effect is removed by using the spectrally useful irradiance for the calculation of the efficiency. In contrast, the double junction exhibits a noticeable functional relationship for the more extreme conditions, while there is a significant amount of scatter occurring closer to the design point. This is due to the variation of environmental conditions at these UFs. The efficiency is, however, reduced for red light, as could be expected from the behaviour of the currents. The effect on the FF is of opposing sign to the effect on the currents, thus it is to some extent offset. However, this only indicates that the secondary spectral effect is not as strong as it would be without the influence of the voltages, and it does not dispute the importance of the primary spectral effect, which was removed before analysing the efficiency. The primary spectral effect is 
directly related to the efficiency, not considering this primary effect would increase the scatter in Fig. 7 very significantly and the shape plotted for the double junction device becomes nearly invisible.



Fig. 7. Influence of UF on the device efficiency.

The magnitude of spectral effects might appear to be small at first glance. They are, however, of the same order as the effects of temperature, irradiance and degradation. It appears from the above discussion that the seasonal variation in the performance can be described exclusively by spectral effects for Loughborough, UK. The device temperatures experienced here are below $65^{\circ} \mathrm{C}$, thus no significant annealing can be expected. Furthermore, the temperature effect for a-Si is known to be very small. The effect of the magnitude of irradiance will possibly be stronger, though apart from this the spectrum appears to be the most important factor. The importance and magnitude of the spectral effect can be seen when extracting the thermal coefficient of the efficiency of a device. These are sometimes reported to be positive [5], but most often the thermal coefficient is negative [6].
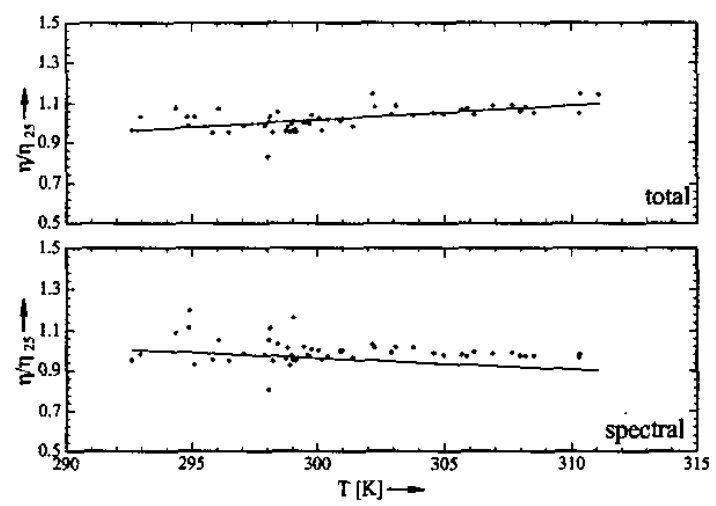

Fig. 8. Perceived thermal coefficient of a double junction when using total and spectral irradiance.

There is an effect of the magnitude of the irradiance on the thermal coefficient, hence it is important to filter measurements to a relatively small bin. Here values between 950 and $1050 \mathrm{~W} / \mathrm{m}^{2}$ are used. A trend line is fitted and all values are presented with respect to the efficiency at $25^{\circ} \mathrm{C}$ in order to ensure comparability between the use of spectral and total irradiance. When considering total irradiance only, the double junction exhibits a positive temperature coefficient. In contrast to this, the device shows a negative temperature coefficient when correcting for the primary spectral effect and using the spectrally useful irradiance only. This illustrates the importance of considering the incident spectrum in any kind of analysis. In the case of investigated devices it appears to be much more significant than the effect of the temperature.

\section{CONCLUSIONS}

The importance of primary spectral effects on the operation of a-Si single and double junction devices is apparent from this investigation. it is clearly shown that double junctions additionally exhibit a significant secondary spectral effect. This effect is important to keep in mind when designing multijunction devices. It can be expected from the above argument that triple junctions are even more prone to spectral variations. Thus a further increase in the number of junctions in one stack might not be desirable. It will increase the efficiency at the design point, i.e. under AM1.5 conditions, but may lead to a poor operational efficiency due to the constant changes in the spectrum.

Having shown the importance of these effects, an effort is now underway to model this behaviour and make these findings applicable to other locations.

\section{ACKNOWLEDGEMENTS}

The authors want to acknowledge the financial support of the Engineering and Physical Sciences Research Council (EPSRC) of the United Kingdom through contract No. GR/N04232. The authors are also grateful for the support of Dr. H. Schade of RWE Solar, Division Phototronics, who supplied the samples.

\section{REFERENCES}

[1] R. Gottschalg, D.G. Infield and M.J. Kearney, "Influence of Environmental Conditions on Outdoor Performance of Thin Film Devices", Seventeenth EU PVSEC, 2001: pp. to be publ.

[2] T.R. Betts, R. Gottschaig and D.G. Infield, "Progress Towards Modelling Solar Spectral Radiation for Optimisation of Amorphous Silicon Photovoltaic Systems", REMIC, 2001, pp. 163-170.

[3] K.A. Emery, "Solar Simulators and I-V Measurement Methods", Solar Cells 18, 1986, pp. 251-260.

[4] R. Rüther, G. Kleiss and K. Reiche, "Spectral Effects on Amorphous Silicon Solar Module Fill Factors", Solar Energy Materials and Solar Cells 71, 2002, pp. 375-385.

[5] D. Carlson, G. Lin and G. Ganguly, "Temperature Dependence of Amorphous Silicon Solar Cell PV Parameters", Twenty-eighth IEEE PVSC, 2000, pp. 707-712.

[6] H. Schade et al., "Application-Related Features of a-Si Based PV Technology", Fifteenth EU PVSEC, 1998: pp. 2054-2057. 